\title{
Changing profiles of pediatric epilepsy surgery candidates over time: A nationwide single-center experience from | 990 to 20 I I
}

\author{
'Herm J. Lamberink, 'Kim Boshuisen, Peter C. van Rijen, Peter H. Gosselaar, and Kees P. J. \\ Braun, on behalf of the Dutch Collaborative Epilepsy Surgery Program (DCESP)
}

Epilepsia, 56(5):717-725, 2015

doi: $10.1111 /$ epi.12974

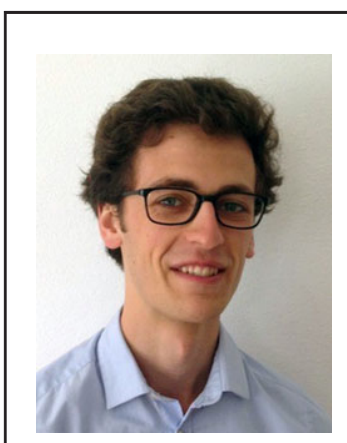

Herm Lamberink is a doctoral student in pediatric neurology at University Medical Center Utrecht.

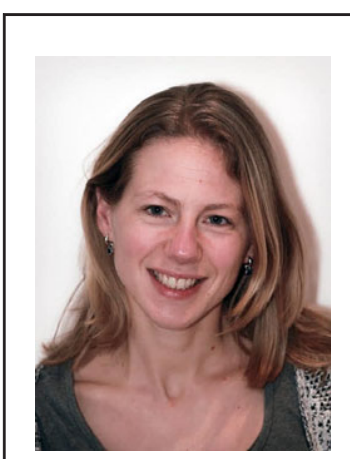

Kim Boshuisen is a resident in neurology and doctoral student in pediatric neurology at University Medical Center Utrecht.

\section{SUMMARY}

Objective: Over the past decades, the number of epilepsy surgeries in children has increased and indications for surgery have broadened. We studied the changes in patient characteristics between 1990 and $201 \mathrm{I}$ in a nationwide cohort and related these to seizure outcome and postoperative medication status. Second, we tried to identify predictors for seizure outcome after pediatric epilepsy surgery.

Methods: To study changes over time, we divided this retrospective cohort of $\mathbf{2 3 4}$ children into two consecutive time periods of II years, and statistically compared the epochs in terms of patient characteristics, surgical variables, complications, seizure outcome, and postoperative medication status. To identify predictors of postoperative seizure freedom, we performed univariable and multivariable logistic regression analyses.

Results: The number of surgeries per year increased from an average of $\mathbf{5}$ in the first, to 16 in the past epoch. Over time, significantly more surgeries were performed for malformations of cortical development, and more patients underwent magnetoencephalography (MEG) and invasive monitoring. Four percent of patients had a serious complication. Complete seizure freedom (Engel class IA) at 2 years after surgery was achieved in $\mathbf{7 4} \%$ of patients, which did not change significantly over time. The proportion of patients who were free from seizures and antiepileptic medication 2 years after surgery significantly increased from $13 \%$ to $32 \%$. Factors predictive of seizure recurrence were preoperative intracranial monitoring, multilobar surgery, etiology, and longer duration of epilepsy before surgery.

Significance: Although more complex cases were operated over time and medication was withdrawn earlier after surgery, success rates at 2 years remained stable. In combination with low complication rates, this underscores the efficacy and safety of pediatric epilepsy surgery. It is important to consider epilepsy surgery early, as longer duration of epilepsy increased the risk of postoperative seizure recurrence. KEY WORDS: Epilepsy, Surgery, Pediatric, Changes in time, Predictors.

Accepted February 18, 2015; Early View publication April 3, 2015

Department of (Child) Neurology and Neurosurgery, Brain Center Rudolf Magnus, University Medical Center Utrecht, Utrecht, The Netherlands

${ }^{1}$ These authors contributed equally to this work.

Address correspondence to Kees P. J. Braun, Department of (Child) Neurology and Neurosurgery, Brain Center Rudolf Magnus, University Medical Center Utrecht, Room KC 03.063.0, PO Box 85090, 3508 AB Utrecht, The Netherlands. E-mail: k.braun@umcutrecht.nl

Wiley Periodicals, Inc.

(C) 2015 International League Against Epilepsy 


\section{H. J. Lamberink et al.}

Epilepsy surgery has become a widely accepted treatment option for children with refractory epilepsy caused by a focal or hemispheric structural epileptogenic source. Between $59 \%$ and $70 \%$ of children become seizure-free after resective surgery. ${ }^{1}$ The ultimate goal of surgery is to "cure" the epilepsy, which can be defined as reaching complete freedom from both seizures and antiepileptic drugs (AEDs) for at least 5 years. ${ }^{2}$

Over the past three decades, the approach to pediatric epilepsy surgery has changed dramatically. Improved imaging techniques with higher field strengths, ${ }^{3}$ specific magnetic resonance imaging (MRI) protocols, and imaging postprocessing in apparent MRI-negative patients have enabled the identification of patients previously not considered eligible for epilepsy surgery. ${ }^{4}$ Other factors that have led to increased numbers of surgeries in children are advances in functional imaging (positron emission tomography/singlephoton emission computed tomography [PET/SPECT]), improved source localization techniques, and intracranial monitoring (grid, stereo-electroencephalography [EEG]). ${ }^{4}$ The past ten years, attitudes toward surgery have changed even more, with the notion that surgery can also be performed in generalized epileptic syndromes when focal or hemispheric MRI abnormalities are present, ${ }^{5-7}$ and the increasing awareness that early surgery (and thus shorter duration of epilepsy) leads to improved seizure and cognitive outcomes. ${ }^{8,9}$

Different studies have shown that the number of patients considered for epilepsy surgery has increased over the years,${ }^{10,11}$ but the number of surgeries has remained stable. ${ }^{11,12}$ In children, however, the number of surgeries has increased with time. ${ }^{11,13}$ Few studies have focused on trends in individual patient characteristics, and the studies that have done so were either in a specific population of patients who underwent temporal lobe epilepsy surgery, ${ }^{14}$ or were single-center studies and therefore carried the risk of presenting selected populations. ${ }^{10,13}$ In only one study was pediatric epilepsy surgery addressed specifically, ${ }^{13}$ whereas the others described populations including all ages. ${ }^{10,14}$

Since 1990, children who are considered candidates for epilepsy surgery in The Netherlands are evaluated during the national multidisciplinary meetings of the Dutch Collaborative Epilepsy Surgery Program (DCESP). When consensus to perform surgery is reached, all children undergo surgery in the University Medical Center Utrecht (UMCU). The number of surgeries has increased rapidly over the past years. Because pediatric epilepsy surgery in our country has been performed in only a single center, this has created the unique opportunity to study characteristics and outcomes of a nationwide patient population over time. The current study provides an overview of pediatric epilepsy surgery in The Netherlands from 1990 to 2011.

Under the assumption that the rising numbers of pediatric epilepsy surgical procedures are paralleled by an increase in complexity of selected cases, we first aimed to explore how patient characteristics, surgical variables, and complication rates changed over two consecutive periods from 19902011. Second, we compared seizure outcome and medication status 2 years after surgery between these epochs. Finally, we identified predictive factors of seizure outcome in the entire surgical cohort.

\section{MeTHODS}

\section{Patients}

This is a retrospective study of all children (aged $<18$ years) who underwent epilepsy surgery in UMCU and who had at least 2 years of follow-up. Patients were included if they underwent resective or disconnective surgery between January 1, 1990 and October 1, 2011. Excluded were patients who underwent palliative procedures (e.g., corpus callosotomy, $\mathrm{n}=16$ ). For patients who were subject to a second surgical procedure $>2$ years later, only the first surgery was evaluated in the current analysis. In total, 234 patients were included.

\section{Data collection}

Data were retrieved from the database of the DCESP and complemented with information from patient records when necessary. Part of this cohort has been included in a previous publication on surgical outcome. ${ }^{15}$ The DCESP receives referrals from all over the country. All results of diagnostic evaluation, partly performed in the referring center, are discussed in the monthly national multidisciplinary meetings, where it is decided whether additional investigations are necessary, and if the patient is eligible for surgery. Over the years, a total of three specialized epilepsy neurosurgeons performed all surgeries.

We collected data on gender, age at onset of epilepsy, age at surgery-including the proportion of children below the age of 2 years - duration of epilepsy, presence of specific electroclinical syndromes reflecting epileptic encephalopathy, presurgical evaluations, side and type of surgery, etiology of epilepsy, and number and type of complications. Seizure outcome was evaluated after 2 years, and expressed as the proportion of patients with Engel class IA (i.e., being completely seizure-free since surgery). ${ }^{16}$ Furthermore, we collected data on antiepileptic drug (AED) use 2 years after surgery. Invasive monitoring included grids, depth electrodes, stereo-EEG recordings or a combination. Type of surgery was classified as "lobar," including frontal, temporal, parietal, and occipital resections or disconnections, "multilobar" resections or disconnections, and peri-insular hemispherectomy, from now "hemispherectomy." Electroclinical syndromes suggesting epileptic encephalopathy were defined according to the 2010 International League Against Epilepsy (ILAE) classification, ${ }^{17}$ including Ohtahara, West, Lennox-Gastaut syndromes, and electrical status epilepticus during sleep/continuous spikes and waves during sleep (ESES/CSWS). Etiology was classified according to 
histopathologic investigation (when available) or MRI. We distinguished "malformations of cortical development" (MCDs) (focal cortical dysplasia, hemimegalencephaly, and polymicrogyria), "tumors" (glioneuronal tumors and lowgrade gliomas), "hippocampal sclerosis," "vascular pathology" (ischemic and hemorrhagic stroke, arteriovenous malformations), "neurocutaneous disorders" (Sturge-Weber syndrome and tuberous sclerosis complex), and "other" (including Rasmussen's encephalitis and unclassifiable pathology).

Complications were classified as either "serious" or "mild and reversible." Serious complications included perioperative death, complications that required invasive treatment, and unforeseen neurologic deficits that persisted for longer than 3 months. Hydrocephalus was reported when occurring at any moment postoperatively (not restricted to the first 3 months) and classified as a serious complication. Mild and reversible complications were classified into four subcategories: (1) surgery-related infections, divided into proven infections (with positive cultures), and suspected infections (but no culture performed) or aseptic inflammatory reactions (negative cultures); (2) non-surgery-related infections such as urinary tract, and upper or lower respiratory tract infections; (3) electrolyte changes for which intervention was started; and (4) transient neurologic deficits that were not anticipated. Foreseen neurologic deficits (i.e., homonymous hemianopia after hemispherectomy) were not considered as true complications.

\section{Study design and statistical analysis}

To study changes over time with regard to preoperative, perioperative, and postoperative variables, the cohort was divided into two epochs of 11 years: children who underwent surgery between 1990 and $2000(n=56)$, and between 2001 and $2011(\mathrm{n}=178)$. The proportions of categorical variables were compared between the two epochs using $\chi^{2}$ statistic, where necessary computed with Fisher's exact testing. For continuous variables, means were compared using a Student's $t$-test or, when data were not normally distributed, Mann-Whitney $U$-test.

To identify predictors of seizure freedom, first univariable logistic regression was performed for all variables included in this study. Variables with a p-value less than 0.10 were then included in a multivariable model. The separate lobar surgery locations were not included in the multivariable regression model because these are subgroups of lobar surgeries already included in the model.

Analyses were performed using IBM SPSS Statistics for Windows version 20.0, (IBM Corp. Armonk, NY, U.S.A.).

\section{RESULTS}

\section{Patient characteristics}

Between 1990 and 2011, 690 children were referred to the DCESP to discuss eligibility for epilepsy surgery

\begin{tabular}{|c|c|c|c|c|}
\hline Variable & $\begin{array}{l}\text { All years } \\
(\mathrm{n}=234)\end{array}$ & $\begin{array}{l}1990-2000 \\
(n=56)\end{array}$ & $\begin{array}{l}200 I-20 I I \\
(n=I 78)\end{array}$ & $\mathrm{p}$-Value \\
\hline $\begin{array}{l}\text { Evaluated patients in } \\
\text { DCESP per year, } \\
\text { mean (SD) }\end{array}$ & $31.4(19.6)$ & $16.9(8.9)$ & $45.8(16.3)$ & $<0.001$ \\
\hline $\begin{array}{l}\text { Surgeries per year, } \\
\text { mean (SD) }{ }^{a}\end{array}$ & $10.6(6.9)$ & $5.1(3.0)$ & $16.2(4.7)$ & $<0.001$ \\
\hline Gender female, \% & 48.3 & 53.6 & 46.6 & 0.37 \\
\hline $\begin{array}{l}\text { Age at seizure onset, } \\
\text { year, median }\end{array}$ & 2.0 & 2.0 & 2.0 & 0.98 \\
\hline $\begin{array}{l}\text { Age at surgery, } \\
\text { year, median }\end{array}$ & 10.4 & 11.4 & 9.4 & 0.48 \\
\hline $\begin{array}{l}\text { Age }<2 \text { years at } \\
\text { surgery, } \%\end{array}$ & 12.8 & 10.7 & 13.5 & 0.59 \\
\hline $\begin{array}{l}\text { Duration of epilepsy } \\
\text { until surgery, year, } \\
\text { median }\end{array}$ & 4.2 & 5.8 & 3.7 & 0.07 \\
\hline Left side, \% & 48.7 & 42.9 & 50.6 & 0.31 \\
\hline $\begin{array}{l}\text { Electroclinical } \\
\text { syndrome, }{ }^{b} \%\end{array}$ & 8.6 & 3.6 & 10.1 & 0.17 \\
\hline West syndrome, \% & 6.0 & 1.8 & 7.3 & \\
\hline $\begin{array}{l}\text { Ohtahara } \\
\text { syndrome, \% }\end{array}$ & 1.7 & 1.8 & 1.7 & \\
\hline ESES/CSWS, \% & 0.9 & 0 & 1.1 & \\
\hline $\begin{array}{l}\text { Lennox-Gastaut } \\
\text { syndrome, } \%\end{array}$ & 0.4 & 0 & 0.6 & \\
\hline Additional diagnostics, ${ }^{c} \%$ & 29.5 & 23.2 & 31.5 & 0.24 \\
\hline $\mathrm{PET}, \%$ & 17.3 & 14.5 & 18.2 & 0.53 \\
\hline MEG, \% & 12.9 & 3.6 & 15.8 & 0.02 \\
\hline SPECT, \% & 5.2 & 12.7 & 2.8 & 0.009 \\
\hline $\begin{array}{l}\text { Intracranial } \\
\text { monitoring, \% }\end{array}$ & 9.4 & 1.8 & 11.8 & 0.03 \\
\hline Etiology & & & & 0.005 \\
\hline MCD, \% & 26.5 & 14.3 & 30.3 & 0.02 \\
\hline Tumor, \% & 34.6 & 37.5 & 33.7 & 0.60 \\
\hline $\begin{array}{l}\text { Hippocampal } \\
\text { sclerosis, \% }\end{array}$ & 14.1 & 19.6 & 12.4 & 0.17 \\
\hline Vascular, \% & 12.4 & 7.1 & 14.0 & 0.17 \\
\hline Neurocutaneous, \% & 7.7 & 8.9 & 7.3 & 0.69 \\
\hline Other, $\%$ & 4.7 & 12.5 & 2.2 & 0.005 \\
\hline \multicolumn{5}{|l|}{ Surgery type } \\
\hline Hemispherectomy, \% & 26.9 & 23.2 & 28.1 & 0.64 \\
\hline Multilobar, \% & 5.6 & 7.1 & 5.1 & \\
\hline Lobar, \% & 67.5 & 69.6 & 66.9 & \\
\hline \multicolumn{5}{|l|}{ Lobar surgery location } \\
\hline Temporal, \% & 70.9 & 74.4 & 69.7 & 0.46 \\
\hline Frontal, \% & 21.5 & 15.4 & 23.5 & \\
\hline Parietal, \% & 5.1 & 5.1 & 5.0 & \\
\hline Occipital, \% & 2.5 & 5.1 & 1.7 & \\
\hline
\end{tabular}

Changes over time compared between two epochs. Bold p-values depict a statistically significant difference between the epochs. Analysis performed with independent samples t-test, Mann-Whitney $U$ test, chi-square or Fisher's exact test when appropriate.

${ }^{a}$ Computed with 234 patients included in this study.

${ }^{b}$ Percentage of patients with one of four categories below. One patient was included with West syndrome and subsequent Lennox-Gastaut syndrome. An additional $2 \%$ of patients had a history of West syndrome (not in table), which resolved into focal seizures; these patients underwent surgery 3 to 12 years later. 'Percentage of patients who underwent one or more of the four diagnostic tests below. One patient can be included in more than one of the categories. DCESP, Dutch Collaborative Epilepsy Surgery Program; ESES, electrical status epilepticus during sleep; CSWS, continuous spikes and waves during sleep: PET, positron emission tomography; MEG, magnetoencephalography; SPECT, single photon emission computed tomography; MCD, malformation of cortical development. 


\section{H. J. Lamberink et al.}

(Table 1). Of these, 234 were operated and fulfilled the inclusion criteria. The proportion of evaluated patients receiving surgery was $47 \%$ between 1990 and 2000, and $48 \%$ between 2000 and $2011(\mathrm{p}=0.94)$. The number of surgeries increased by a factor of three over time; from an average of five surgeries per year in the first epoch, to an average of 16 in the last $(\mathrm{p}<0.001)$. The median duration between epilepsy onset and surgery decreased with 2.1 years, from 5.8 years in the first epoch to 3.7 in the last, although this difference was not statistically significant $(p=0.07)$. Age, gender, and side of surgery did not differ between the two epochs. The contribution of the different etiologies changed significantly over time $(p=0.03)$, with a relative increase of patients with malformation of cortical development and a decrease in "other pathologies." Nine percent of children had a clearly described electroclinical syndrome reflecting epileptic encephalopathy at time of surgery. A threefold increase in the proportion of these patients was seen over time, although not significantly.

\section{Preoperative additional investigations}

Supplementary to standard diagnostic examinationsincluding EEG and MRI-30\% of the patients needed additional diagnostic testing to further localize the epileptogenic source (Table 1). These included magnetoencephalography (MEG), PET, SPECT, or intracranial monitoring with subdural grids, depth electrodes, stereo-EEG, or a combination. MEG was used more frequently over time, from $4 \%$ to $16 \%$ $(p=0.02)$, whereas the number of patients evaluated with SPECT has decreased from $13 \%$ to $3 \%(\mathrm{p}=0.009)$. We did, however, perform SPECT registrations in the latter epoch and are using it more frequently in current practice, but in the presented cohort patients evaluated by SPECT have not been found eligible for surgery. The proportion of invasive recordings increased significantly from $2 \%$ to $12 \%$ of patients.

\section{Surgical procedures}

Twenty-seven percent of the surgeries were hemispherectomies, 6\% were multilobar resections, and 68\% lobar resections or disconnections (Table 1). Types of surgery did not change over time. Of the lobar surgeries, $71 \%$ were temporal. The number of frontal surgeries increased, although not significantly.

\section{Complications}

Four percent of all patients had a serious complication (Table 2). The proportion of serious and mild or reversible complications remained stable over the years $(\mathrm{p}=1.00$ and $\mathrm{p}=0.71$, respectively). From the patients with a serious complication directly related to surgery, only one $(0.4 \%)$ developed a permanent hemiplegia as result of an ischemic lesion after an insular resection. Eight patients $(3.5 \%)$ had to undergo invasive treatment for several complications, including intracranial hema-

\begin{tabular}{|c|c|c|c|c|}
\hline Variable & $\begin{array}{l}\text { All years } \\
(\mathrm{n}=230)\end{array}$ & $\begin{array}{l}1990-2000 \\
(\mathrm{n}=55)\end{array}$ & $\begin{array}{l}200|-20| I \\
(n=\mid 75)\end{array}$ & $\begin{array}{l}\mathrm{P}- \\
\text { Value }\end{array}$ \\
\hline Serious complications, \% & 3.9 & 3.6 & 4.0 & 1.00 \\
\hline Invasive treatment, \% & 3.5 & 3.6 & 3.3 & \\
\hline Hydrocephalus, \% & 1.3 & 1.8 & I.I & \\
\hline $\begin{array}{l}\text { Permanent } \\
\text { neurologic deficit, \% }\end{array}$ & 0.4 & 0 & 0.6 & \\
\hline Death, \% & 0 & 0 & 0 & \\
\hline $\begin{array}{l}\text { Mild and reversible } \\
\text { complications, } \%\end{array}$ & 14.8 & 16.4 & 14.3 & 0.71 \\
\hline $\begin{array}{l}\text { Infections, surgery } \\
\text { related, \% }\end{array}$ & 4.7 & 5.4 & 4.6 & \\
\hline Documented, \% & 0.4 & 1.8 & 0 & \\
\hline Suspected/aseptic, \% & 4.3 & 3.6 & 4.6 & \\
\hline $\begin{array}{l}\text { Infections, } \\
\text { non-surgery-related, \% }\end{array}$ & 5.2 & 7.3 & 4.6 & \\
\hline $\begin{array}{l}\text { Transient neurologic } \\
\text { deficits, } \%^{a}\end{array}$ & 1.7 & 1.8 & 1.7 & \\
\hline $\begin{array}{l}\text { Intervention for } \\
\text { electrolyte change, \% }\end{array}$ & 3.5 & 1.8 & 4.0 & \\
\hline \multicolumn{5}{|c|}{$\begin{array}{l}\text { Complications compared between two epochs, divided between serious, } \\
\text { and mild and reversible complications. A patient could be included in more } \\
\text { than one category. Analysis was performed with chi-square or Fisher's exact } \\
\text { test when appropriate. } \\
{ }^{a} \text { Defined as resolving within } 3 \text { months after surgery. }\end{array}$} \\
\hline
\end{tabular}

toma, increased intracranial pressure, cerebrospinal fluid (CSF) leakage, and hydrocephalus. No deaths occurred as a complication of surgery. Within the follow-up period, two patients died: one of pulmonary failure during intensive care treatment for refractory epilepsy 3 months after surgery, and one at 6 months after surgery of unknown cause.

Mild complications included mainly infections or suspected infections $(9.9 \%)$. From the group of surgery-related possible infections, a positive CSF culture was identified in only $0.4 \%$. The remaining surgery-related presumed infections $(4.3 \%)$ where treated with antibiotics even despite negative cultures. The non-surgery-related infections (4.7\%) consisted of respiratory tract infections, urinary tract infections and gastroenteritis. Two percent of the patients had unforeseen neurologic deficits, which normalized within the first 3 months after surgery, and $3.5 \%$ of the patients received treatment for electrolyte disturbances after surgery.

\section{Surgical outcome}

Seizure outcome remained stable over the two epochs $(\mathrm{p}=0.09)$, with $81 \%$ of the patients being free from disabling seizures (Engel class $\mathrm{I}^{16}$ ) after 2 years, and $74 \%$ with complete seizure freedom since surgery (Engel class IA) (Table 3). Eight percent had rare disabling seizures (Engel class II). In total, approximately $89 \%$ of the patients had a clear benefit from surgery (Engel class I and II), 6\% had worthwhile improvement (Engel class III), and 6\% did not have any improvement (Engel class IV). The proportion of 
Table 3. Trends in time-surgical outcome

\begin{tabular}{|c|c|c|c|c|}
\hline Variable & $\begin{array}{l}\text { All years } \\
(\mathrm{n}=234)\end{array}$ & $\begin{array}{l}1990-2000 \\
(n=56)\end{array}$ & $\begin{array}{l}200 I-20 I I \\
(n=I 78)\end{array}$ & $\mathrm{p}$-Value \\
\hline $\begin{array}{l}\text { Completely seizure- } \\
\text { free (SF), } 2 \text { year, \% }\end{array}$ & 74.4 & 71.4 & 75.3 & 0.57 \\
\hline \multicolumn{5}{|l|}{$\begin{array}{l}\text { Engel classification, } \\
2 \text { year, \% }\end{array}$} \\
\hline Class I & 81.2 & 78.6 & 82.0 & 0.09 \\
\hline Class II & 7.7 & 5.4 & 8.4 & \\
\hline Class III & 5.6 & 12.5 & 3.4 & \\
\hline Class IV & 5.6 & 3.6 & 6.2 & \\
\hline $\begin{array}{r}\text { AED-free, } \\
2 \text { year, } \%\end{array}$ & 28.8 & 14.3 & 33.3 & 0.006 \\
\hline $\begin{array}{l}\text { SF and AED-free, } \\
2 \text { year, \% }\end{array}$ & 27.0 & 12.5 & 31.6 & 0.005 \\
\hline \multicolumn{5}{|c|}{$\begin{array}{l}\text { Trends in time in seizure outcome and medication status at } 2 \text { years after sur- } \\
\text { gery. Bold p-values depict a statistically significant difference between the } \\
\text { epochs. Analysis was performed with } \chi^{2} \text { or Fisher's exact test when appropriate. } \\
\text { SF, complete seizure freedom at } 24 \text { months (Engel class IA); AED, antiepi- } \\
\text { leptic drug. }\end{array}$} \\
\hline
\end{tabular}

patients for whom AEDs were completely withdrawn within 2 years after surgery has significantly increased, from $14 \%$ to $33 \%(p=0.006)$. Of all patients who were off antiepileptic drugs, 94\% had been completely seizure-free since surgery.

In patients with a follow-up of at least 5 years $(n=136$, median follow-up 8 years), the proportion of patients with an Engel class I or IA score at latest follow-up was $75 \%$ and $65 \%$, respectively (Table S1). The proportion of seizureand AED-free patients was 55\%. No significant changes over time were observed. The median follow-up duration of patients operated during the first time epoch, however, was significantly longer than of those from the second epoch $(\mathrm{p}<0.001)$. We examined possible relations between follow-up duration and the three long-term outcome measures using logistic regression. There was no significant relation between follow-up duration in years and Engel class I (odds ratio [OR] 0.98), Engel class IA (OR 1.00), or seizure and AED freedom (OR 1.05), at latest follow-up $(\mathrm{p}=0.76$, $\mathrm{p}=0.97$, or $\mathrm{p}=0.39$, respectively).

\section{Predictors of outcome}

We analyzed which factors were associated with complete seizure freedom (Engel class IA) (Table 4). In multivariable analysis, multilobar surgery $(\mathrm{OR} 0.18, \mathrm{p}=0.02)$, presurgical invasive monitoring $(\mathrm{OR} 0.28, \mathrm{p}=0.02)$, and

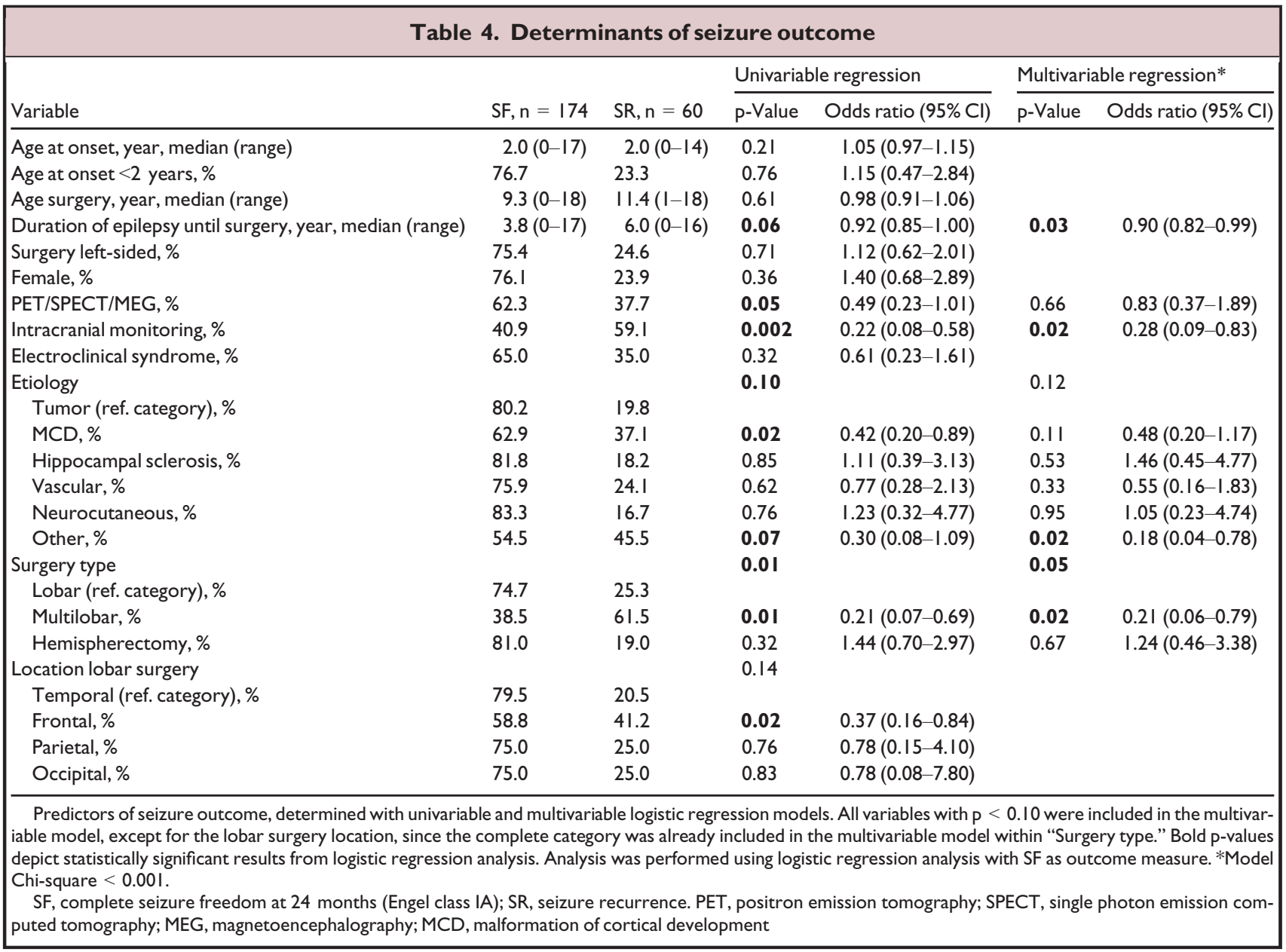


longer duration of epilepsy until surgery (OR 0.90 per year, $\mathrm{p}=0.03$ ) were independently associated with unfavorable seizure outcome (not reaching Engel class IA). Etiology was also related with outcome, with "other etiology" (OR $0.18, \mathrm{p}=0.02$ ) being an independent predictor of seizure recurrence.

\section{Discussion}

In this Dutch pediatric epilepsy surgery cohort, operated between 1990 and 2011, we studied patient and surgery characteristics to identify changes over time. We found several shifts in patient characteristics. The unselected and nationwide nature of this cohort provides a unique opportunity to study the evolving field of pediatric epilepsy surgery. Over the past two decades, the number of evaluated children for surgery and the number of surgeries increased significantly, with more operations on MCDs, and an increased proportion of preoperative MEG recordings and invasive monitoring. The complication rate was low and stable over time. Seizure outcomes at 2 years remained stable, with $90 \%$ of patients having achieved improvement in epilepsy severity and $74 \%$ being completely seizure-free since surgery. Over time, the interval to start of AED withdrawal shortened, resulting in significantly increased AED freedom at 2 years after surgery. Multilobar surgery, presurgical invasive monitoring, etiology, and longer duration of epilepsy independently predicted poor seizure outcome.

\section{Patient characteristics}

The relative increase in the number of patients with MCD, presence of an electroclinical syndrome of epileptic encephalopathy, and the increased proportion of patients with preoperative invasive monitoring, reflect a shift toward more complex cases. In a population of all ages, a similar trend toward more complex cases was observed with less resections on benign tumors and more on patients with focal cortical dysplasia. ${ }^{10}$ In our cohort, the number of frontal surgeries increased, although not significantly.

With regard to etiology, postoperative seizure freedom rates of children with malformations of cortical development, particularly those with focal cortical dysplasia (FCD) type I, ${ }^{18}$ are known to be lower than those of patients with other pathologies. Chern et al. ${ }^{19}$ reported a seizure freedom rate of $60 \%$ (range $46-70 \%$ ) for patients with FCD, compared to $71 \%$ (range $36-100 \%$ ) for tumor patients. ${ }^{20}$ In the current study, this lower seizure freedom rate for MCD patients was also found, with $63 \%$ of the patients reaching complete seizure freedom compared to success rates over $80 \%$ for tumor, hippocampal sclerosis, and vascular etiology. The changes in resection type and identified etiologies indicate a clear shift in the utilization of pediatric epilepsy surgery. At the time when many pediatric epilepsy surgery programs started, mainly children with well-circumscribed focal, MRI-visible, and preferably temporal structural lesions were considered eligible candidates for surgical treatment. Over the years, indications for surgery have now broadened, including children with frontal or posterior quadrant epilepsies, generalized epileptic encephalopathies with focal or hemispheric structural pathology, ${ }^{5-7}$ and MRInegative cases. $3,4,10$

The trends in the number of specific additional presurgical investigations support the hypothesis that more complex cases are operated in the latest years: the increased use of MEG and invasive monitoring are indicative of the need for more precise localization of the epileptogenic source. In our nationwide single-center experience, the availability of acute corticography since 2000, of 3T MRI since 2004, and of neuronavigation since 2003, improved diagnostic accuracy and the ability to delineate the epileptogenic zone. Despite the trend toward the selection of more complex cases, with inherent lower a priori probability of reaching seizure control, seizure outcomes were shown to remain remarkably constant over time, with around $75 \%$ of complete seizure freedom at 2 years. This likely reflects the team's learning curve, experience, and access to diagnostic procedures.

\section{AED withdrawal policy}

AED freedom rates in our cohort increased from $14 \%$ to $33 \%$, reflecting a more aggressive AED withdrawal policy, which seems to be a more global phenomenon; in a survey regarding AED withdrawal policy of $2007,71 \%$ of U.S. epileptologists would wait for at least 2 years of postoperative seizure freedom before considering discontinuation of drugs, and some of these would even never consider withdrawal of AEDs. ${ }^{21}$ In $2012^{22}$ and $2013,{ }^{23}$ only $3-7 \%$ of U.S. and Canadian epileptologists would wait to withdraw AEDs for at least 2 years in patients receiving polytherapy and 24$35 \%$ would wait 2 years for patients receiving monotherapy, reflecting a shift in postoperative drug withdrawal policy.

The proportion of patients who are ultimately AED free is different between children and adults, as shown in a Swedish study on long-term postoperative outcomes: 10 years after surgery, $86 \%$ of the children had discontinued AEDs, compared to $43 \%$ of adults. ${ }^{24}$ The timing of AED withdrawal, however, seems to be similar in children and adults: a meta-analysis including both adults and children reported an average time between surgery and initiation of AED withdrawal of 14 months, and the average time to complete discontinuation of 30 months. ${ }^{25}$ The TimeToStop study on 766 children revealed that in children the median time to start reducing drugs was 12.5 months, and the median time to discontinuation was 28.8 months. ${ }^{26}$ In that study, early AED withdrawal was thought to unmask surgical failure sooner, but not at the cost of long-term outcomes. This was recently confirmed in a mainly adult cohort study. ${ }^{27}$ The changes in survey responses point toward a trend of earlier AED withdrawal, similar to what we observed in the current study. In contrast to these findings, a study from the pediatric UCLA cohort ${ }^{13}$ attributed an 
increased seizure freedom rate in recently operated children to longer AED use after surgery, as compared to children who underwent surgery in earlier time epochs.

\section{Predictors of outcome}

Longer duration of epilepsy, multilobar surgery, etiology, and intracranial presurgical monitoring were found to be independently associated with unfavorable seizure outcome. Other known factors predicting seizure recurrence in pediatric epilepsy surgery include extratemporal resections, multifocal MRI lesions, incomplete resection of the lesion, and the presence of presurgical generalized tonic-clonic seizures. ${ }^{1}$

Intracranial monitoring has previously been reported to predict unfavorable postoperative seizure outcome. ${ }^{28}$ The predictive value of intracranial monitoring, however, may highly depend on the population, since other studies have reported no or an inverse relation between invasive monitoring and seizure outcomes. ${ }^{29-31}$

Longer duration of epilepsy until surgery was associated with unfavorable seizure outcome, confirming findings from previous studies..$^{20,24,32,33}$ In addition, it has been demonstrated previously that longer duration of epilepsy has a negative impact on cognitive change after surgery. ${ }^{1,34} \mathrm{We}$ may expect that the recent evidence on the beneficial cognitive effects of early epilepsy surgery lead to a further decrease in age at surgery in future pediatric cohorts.

\section{Complications}

The risk of serious surgical complications was $4 \%$, with only $0.4 \%$ resulting in permanent neurologic damage. The frequency of mild complications was higher, but it should be acknowledged that not all presumed surgical complications are true complications requiring therapy. In some children, antibiotic treatment was started when infection was suspected, but only in a minority was direct proof of infection by positive culture obtained. After pediatric hemispherectomy, for example, fever, increased peripheral white blood cell count, and CSF protein are frequently observed, but this is rarely caused by intracranial infection. ${ }^{35}$ Changes in serum sodium levels in some children led to treatment (fluid restriction or sodium supplementation) without direct proof of syndrome of inappropriate antidiuretic hormone secretion (SIADH) or cerebral salt wasting; the electrolyte disbalance may have also been caused by specific AEDs (e.g., carbamazepine). If we do not take above-mentioned "mild complications" into account, only $2.1 \%$ of the patients had a mild surgery-related complication (documented infections and transient neurological deficits).

Several studies have used different criteria to report their complication rates. A recent review on complications after epilepsy surgery reported $5.7 \%$ minor and $4.5 \%$ major medical complications, and $11.2 \%$ minor and $5.1 \%$ major neurologic complications for children. ${ }^{36}$ The higher complication rate in that study can be explained by the authors' choice to include anticipated neurologic deficits that are inherent to surgery. Hemb et al. ${ }^{13}$ have reported serious and permanent complications only, with a complication rate of $9.2 \%$. The difference in complication rate between their and our cohort can be explained by the much higher percentage of hemispherectomy patients in their cohort and the difference in definition of complications that included long-term deaths unrelated to surgery in the cohort of Hemb et al., whereas we chose not to consider those cases.

The 22 children who underwent invasive EEG monitoring preoperatively did not have any complication related to this procedure. Studies in larger cohorts have concluded that invasive monitoring bears limited risks, and large benefits, ${ }^{37,38}$ with a risk for major or permanent complications of $0.6 \% .^{36}$

\section{Strengths and limitations}

The current study distinguishes itself from previous studies because of its nationwide setup. The population included all children who underwent epilepsy surgery in The Netherlands between 1990 and 2011, and therefore the observed trends are truly reflective of changes in pediatric epilepsy surgery in an unbiased cohort, although we acknowledge that there will be differences between countries and continents.

The study also has several limitations. First, no data are presented on changes in preoperative evaluations and diagnostics for children who were referred but eventually rejected for epilepsy surgery. There was an increasing number of patients referred to the DCESP, which reflects the increased knowledge of the benefits of epilepsy surgery among physicians taking care of children with refractory epilepsy.

Second, the follow-up period of 2 years after surgery is relatively short. When our success rate is compared to other cohorts, the proportion of completely seizure-free patients of $74 \% 2$ years after surgery is high, since the average postoperative seizure freedom rate in children is reported to be 59-70\%. ${ }^{1}$ However, seizure-freedom rates are known to decline over the years ${ }^{39}$ and seem to remain stable after 5 years postoperatively. ${ }^{24}$ For 136 of our patients, followup data after at least 5 years was available, revealing a relatively high proportion of patients with Engel class IA (65\%), with no differences between the two epochs. However, a direct comparison of long-term outcome between the two time periods is complicated by the inherent differences in follow-up duration.

\section{Conclusion}

The outcome of pediatric epilepsy surgery in The Netherlands has been stable over time with high success rates and low complication rates. Although we found a shift toward surgery in more complex patient categories (such as MCDs), and a tendency toward more complex lesion locations (such as frontal resections), seizure-freedom rates remained steadily high, and the number of children who reached both freedom from seizures and AEDs sig- 
nificantly increased over the years. Predictors independently associated with unfavorable seizure outcome were multilobar surgery, invasive monitoring, and longer epilepsy duration. Unfavorable seizure outcomes with longer duration of epilepsy underscore the fact that early surgery should be the goal. The high seizure freedom and low complication rates in this nationwide cohort reflect the advantage of a centralized multidisciplinary assessment of surgical candidates, and the performance of surgery in highly specialized medical centers.

\section{ACKnOWLedgments}

We gratefully acknowledge Dr. C.S.P. Uiterwaal and Dr. F.S.S. Leijten for their valuable comments.

\section{Disclosure}

None of the authors have any conflict of interest to disclosure. The authors have read the Journal's guidelines for ethical publication, and declare that the work described is consistent with these guidelines.

\section{REFERENCES}

1. Spencer S, Huh L. Outcomes of epilepsy surgery in adults and children. Lancet Neurol 2008;7:525-537.

2. Schmidt D, Baumgartner C, Löscher W. The chance of cure following surgery for drug-resistant temporal lobe epilepsy. What do we know and do we need to revise our expectations? Epilepsy Res 2004;60:187-201.

3. Phal PM, Usmanov A, Nesbit GM, et al. Qualitative comparison of 3$\mathrm{T}$ and 1.5-T MRI in the evaluation of epilepsy. AJR Am J Roentgenol 2008:191:890-895.

4. So EL, Lee RW. Epilepsy surgery in MRI-negative epilepsies. Curr Opin Neurol 2014;27:206-212.

5. Wyllie E, Lachhwani DK, Gupta A, et al. Successful surgery for epilepsy due to early brain lesions despite generalized EEG findings. Neurology 2007;69:389-397.

6. Lachhwani DK. Pediatric epilepsy surgery: lessons and challenges. Semin Pediatr Neurol 2005;12:114-118.

7. Boshuisen K, Schooneveld MM, Leijten FSS, et al. Contralateral MRI abnormalities affect seizure and cognitive outcome after hemispherectomy. Neurology 2010;75:1623-1630.

8. Freitag H, Tuxhorn I. Cognitive function in preschool children after epilepsy surgery: rationale for early intervention. Epilepsia 2005; 46:561-567

9. Lettori D, Battaglia D, Sacco A, et al. Early hemispherectomy in catastrophic epilepsy: a neuro-cognitive and epileptic long-term follow-up. Seizure 2008; 17:49-63.

10. Bien CG, Raabe AL, Schramm J, et al. Trends in presurgical evaluation and surgical treatment of epilepsy at one centre from 19882009. J Neurol Neurosurg Psychiatry 2013;84:54-61.

11. Schiltz NK, Koroukian SM, Lhatoo SD, et al. Temporal trends in pre-surgical evaluations and epilepsy surgery in the U.S. from 1998 to 2009. Epilepsy Res 2013;103:270-278.

12. Englot DJ, Ouyang D, Garcia PA, et al. Epilepsy surgery trends in the United States, 1990-2008. Neurology 2012;78:1200-1206.

13. Hemb M, Velasco TR, Parnes MS, et al. Improved outcomes in pediatric epilepsy surgery: the UCLA experience, 1986-2008. Neurology 2010;74:1768-1775.

14. Helmstaedter C, May TW, von Lehe M, et al. Temporal lobe surgery in Germany from 1988 to 2008: diverse trends in etiological subgroups. Eur J Neurol 2014;21:827-834.

15. Van Oijen M, De Waal H, Van Rijen PC, et al. Resective epilepsy surgery in childhood: the Dutch experience 1992-2002. Eur J Paediatr Neurol 2006;10:114-123.
16. Engel JJ, Van Ness PC, Rasmussen TB, et al. Outcome with respect to epileptic seizures. In Engel J Jr (Ed) Surgical treatment of the epilepsies. New York, NY: Raven Press, 1993:609-621.

17. Berg AT, Berkovic SF, Brodie MJ, et al. Revised terminology and concepts for organization of seizures and epilepsies: report of the ILAE Commission on Classification and Terminology, 2005-2009. Epilepsia 2010;51:676-685.

18. Krsek P, Pieper T, Karlmeier A, et al. Different presurgical characteristics and seizure outcomes in children with focal cortical dysplasia type I or II. Epilepsia 2009;50:125-137.

19. Chern JJ, Patel AJ, Jea A, et al. Surgical outcome for focal cortical dysplasia: an analysis of recent surgical series. $J$ Neurosurg Pediatr 2010;6:452-458.

20. Englot DJ, Berger MS, Barbaro NM, et al. Predictors of seizure freedom after resection of supratentorial low-grade gliomas. A review. J Neurosurg 2011;115:240-244.

21. Berg AT, Langfitt JT, Spencer SS, et al. Stopping antiepileptic drugs after epilepsy surgery: a survey of US epilepsy center neurologists. Epilepsy Behav 2007;10:219-222.

22. Téllez-Zenteno JF, Ronquillo LH, Jette N, et al. Discontinuation of antiepileptic drugs after successful epilepsy surgery - a Canadian Survey. Epilepsy Res 2012;102:23-33.

23. Swisher CB, Sinha SR. Survey of current practices among US epileptologists of antiepileptic drug withdrawal after epilepsy surgery. Epilepsy Behav 2013;26:203-206.

24. Edelvik A, Rydenhag B, Olsson I, et al. Long-term outcomes of epilepsy surgery in Sweden - a national prospective and longitudinal study. Neurology 2013;81:1244-1251.

25. Ladino LD, Hernandez-Ronquillo L, Tellez-Zenteno JF. Management of antiepileptic drugs following epilepsy surgery: a meta-analysis. Epilepsy Res 2014;108:765-774.

26. Boshuisen K, Arzimanoglou A, Cross JH, et al. Timing of antiepileptic drug withdrawal and long-term seizure outcome after paediatric epilepsy surgery (TimeToStop): a retrospective observational study. Lancet Neurol 2012; 11:784-791.

27. Yardi R, Irwin A, Kayyali $\mathrm{H}$, et al. Reducing versus stopping antiepileptic medications after temporal lobe surgery. Ann Clin Transl Neurol 2014;1:115-123.

28. Tonini C, Beghi E, Berg AT, et al. Predictors of epilepsy surgery outcome: a meta-analysis. Epilepsy Res 2004;62:75-87.

29. Kumar A, Valentin A, Humayon D, et al. Preoperative estimation of seizure control after resective surgery for the treatment of epilepsy. Seizure 2013;22:818-826.

30. von der Brelie C, Malter MP, Niehusmann P, et al. Surgical management and long-term seizure outcome after epilepsy surgery for different types of epilepsy associated with cerebral cavernous malformations. Epilepsia 2013;54:1699-1706.

31. Elsharkawy AE, Behne F, Oppel F, et al. Long-term outcome of extratemporal epilepsy surgery among 154 adult patients. J Neurosurg 2008;108:676-686.

32. Simasathien T, Vadera S, Najm I, et al. Improved outcomes with earlier surgery for intractable frontal lobe epilepsy. Ann Neurol 2013;73:646-654

33. Englot DJ, Chang EF. Rates and predictors of seizure freedom in resective epilepsy surgery: an update. Neurosurg Rev 2014;37:389-405.

34. Van Schooneveld MM, Braun KP. Cognitive outcome after epilepsy surgery in children. Brain Dev 2013;35:721-729.

35. Phung J, Mathern GW, Krogstad P. Timing and predictors of fever and infection after craniotomy for epilepsy in children. Pediatr Infect Dis $J$ 2013;32:450-459.

36. Hader WJ, Téllez-Zenteno JF, Metcalfe A, et al. Complications of epilepsy surgery: a systematic review of focal surgical resections and invasive EEG monitoring. Epilepsia 2013;54:840-847.

37. Hedegard E, Bjellvi J, Edelvik A, et al. Complications to invasive epilepsy surgery workup with subdural and depth electrodes: a prospective population-based observational study. J Neurol Neurosurg Psychiatry 2014;85:716-720.

38. Wellmer J, von der Groeben F, Klarmann U, et al. Risks and benefits of invasive epilepsy surgery workup with implanted subdural and depth electrodes. Epilepsia 2012;53:1322-1332.

39. de Tisi J, Bell GS, Peacock JL, et al. The long-term outcome of adult epilepsy surgery, patterns of seizure remission, and relapse: a cohort study. Lancet 2011;378:1388-1395. 


\section{Appendix I}

\section{Dutch Collaborative Epilepsy Surgery Program}

Current and former* members:

\section{University Medical Center Utrecht}

K.P.J. Braun, F.E. Jansen, K. Geleijns, O van Nieuwenhuizen* (pediatric neurologists); F.S.S. Leijten, C.H. Ferrier, A.C. van Huffelen* (clinical neurophysiologists); P.C. van Rijen, P. Gosselaar, C.W. van Veelen* (neurosurgeons); G.A. de Kort (neuroradiologist); M.M. van Schooneveld, O. Braams, M.J. van Zandvoort (neuropsychologists); W.T. Spliet (neuropathologist); T.A. Gebbink (physician assistant); J.M. Ophorst-van Eck (specialized nurse); T. De Groot (secretary).

\section{Epilepsy Centers of the Netherlands Foundation (SEIN)}

R.M. Debets* (neurologist, adult epileptologist); D. Velis, S. Claus, M. Bourez-Swart, A.W. de Weerd, W. van Emde Boas* (clinical neurophysiol- ogists, epileptologists); W.C. Alpherts* (neuropsychologist); A. Hospes (specialized nurse).

\section{Epilepsy Center Kempenhaeghe}

A.J. Colon, L. Wagner, H.W. ter Spill*, E.P. Veltman* (clinical neurophysiologists, epileptologists).

\section{Free University Medical Center Amsterdam}

J.C. Baayen (neurosurgeon); C.J. Stam, H.E. Ronner (clinical neurophysiologists); E. Comans (nuclear radiologist); E. Sanchez (neuroradiologist).

\section{Maastricht University Medical Center}

J. Dings (neurosurgeon); V. van Kranen-Mastenbroek (clinical neurophysiologist); P. Hofman (neuroradiologist).

\section{SUPPORTING INFORMATION}

Additional Supporting Information may be found in the online version of this article:

Table S1. Long-term follow-up. 